\title{
Enfermedad de Kawasaki: ¿una entidad exclusiva de niños?
}

\author{
Kawasaki disease, is it exclusive of children? \\ Yesid A. Saavedra-González ${ }^{1 *}$, Miguel Vega-Arango², Carlos A. Arboleda-Vallejo3, \\ Roberto Parga-Gómez y Pedro Abad-Díaz ${ }^{5}$
}

${ }^{1}$ Servicio de Cardiología Clínica, Universidad de Antioquia; ${ }^{2}$ Departamento de Radiología, Universidad CES; ${ }^{3}$ Departamento de Radiología, Universidad de Antioquia; ${ }^{4}$ Sección de Cardiología, Clínica Las Vegas; ${ }^{5}$ Servicio de Dinámica, Instituto de Alta Tecnología Médica (IATM). Medellín, Colombia

\begin{abstract}
Resumen
La enfermedad de Kawasaki es un síndrome febril agudo y autolimitado, de presunta etiología autoinmune, que característicamente afecta a niños menores de 5 años. Esta enfermedad consiste en una vasculitis aguda de pequeños y medianos vasos que favorece la formación de aneurismas en las arterias coronarias. El diagnóstico en la fase aguda es esencialmente clínico, mientras que en la fase crónica suele hacerse al documentar las secuelas vasculares. La importancia de la identificación apropiada de esta enfermedad radica en que muchos de los infartos fatales y no fatales en personas menores de 40 años tienen hallazgos compatibles con este diagnóstico. Se presenta el caso de un adulto joven con dolor torácico y angiotomografía coronaria con hallazgos compatibles con secuelas de enfermedad de Kawasaki del adulto.
\end{abstract}

Palabras clave: Angiotomografía. Aneurisma coronario. Enfermedad de Kawasaki. Dolor torácico.

\begin{abstract}
Kawasaki disease is an acute and self-limited febrile syndrome with a presumed autoimmune etiology that characteristically affects children under 5 years of age. Kawasaki disease is an acute vasculitis of small and medium vessels that facilitates the formation of coronary aneurysms. The diagnosis of the disease during the acute phase is essentially clinical, while the diagnosis of Kawasaki disease during the chronic phase is performed when the vascular complications are detected. The importance of proper identification of Kawasaki disease lies in the fact that many of the fatal and non-fatal infarcts in young adults (<40 years) have findings compatible with this diagnosis. This article describes the case of a young patient with chest pain and coronary angiotomography showing sequelae of Kawasaki disease in the adult.
\end{abstract}

Key words: Angiotomography. Coronary aneurysm. Kawasaki disease. Chest pain.

\section{Caso}

Paciente masculino, de 25 años, quien consultó a Cardiología por cuadro clínico de tres meses de evolución, consistente en dolor torácico retroesternal, opresivo, no irradiado, que se desencadenaba con el ejercicio y mejoraba con el reposo. No tenía antecedentes personales conocidos, y previo al evento actual era completamente asintomático con un alto nivel de actividad física. El examen físico inicial fue normal.

\section{Correspondencia:}

*Yesid A. Saavedra-González

E-mail: yesaago@gmail.com;

yesaago@hotmail.com
Fecha de recepción: 30-01-2019

Fecha de aceptación: 19-05-2020

DOI: 10.24875/RCCAR.M21000044
Disponible en internet: 10-06-2021 Rev Colomb Cardiol. 2021;28(2):175-179 www.rccardiologia.com 0120-5633 @ 2020 Sociedad Colombiana de Cardiología y Cirugía Cardiovascular. Publicado por Permanyer. Este es un artículo open access bajo la licencia CC BY-NC-ND (http://creativecommons.org/licenses/by-nc-nd/4.0/). 


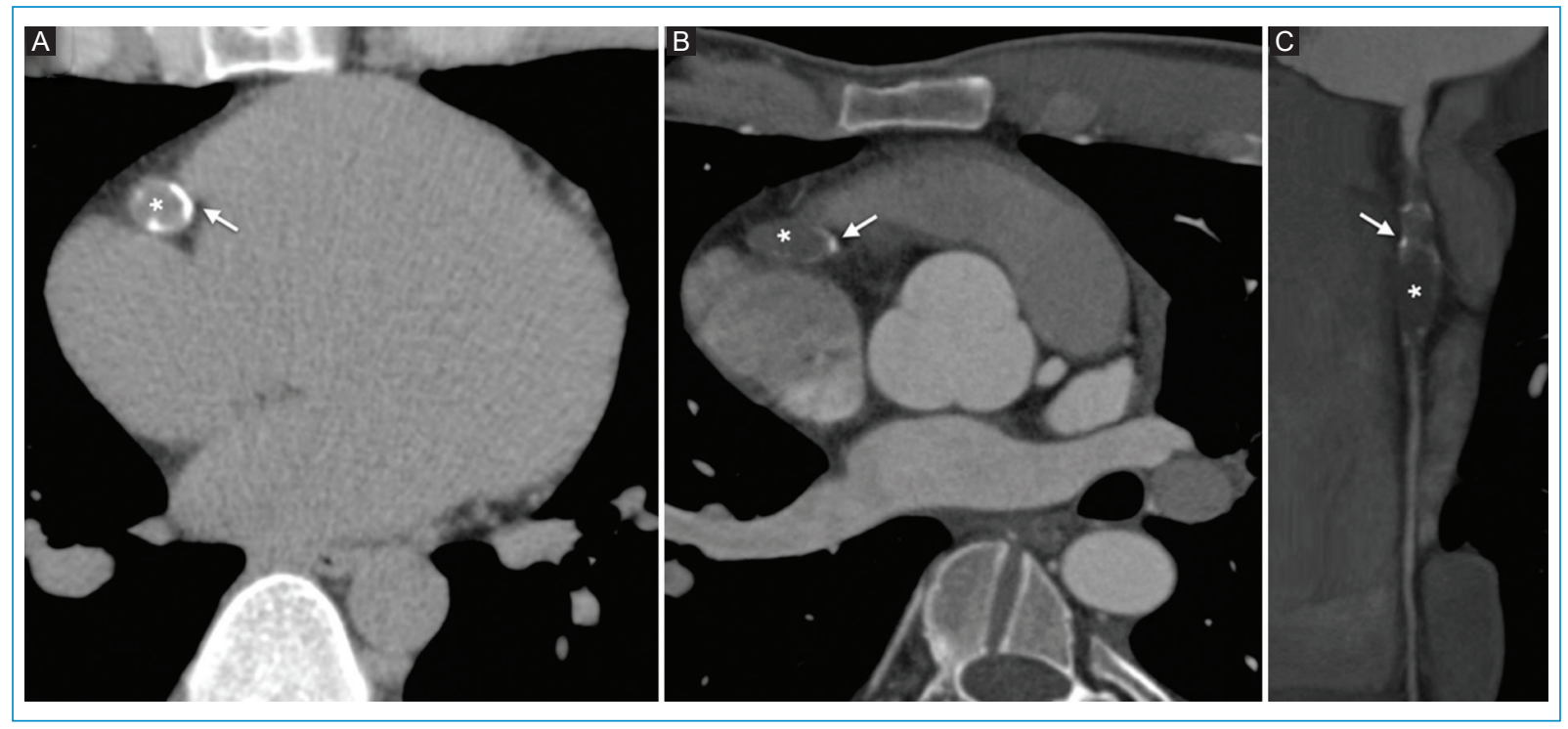

Figura 1. Angiotomografía de arterias coronarias. A: fase simple en la que se observa aneurisma de la ACD con calcificaciones periféricas (flechas). B: fase angiográfica en la que aprecia trombo hipodenso en el interior del aneurisma (asteriscos). C: reconstrucción curva que demuestra la extensión longitudinal del aneurisma (11 mm), las calcificaciones periféricas (flechas), el trombo oclusivo organizado en su interior (asterisco) y el llenado distal a través de circulación colateral heterocoronaria.

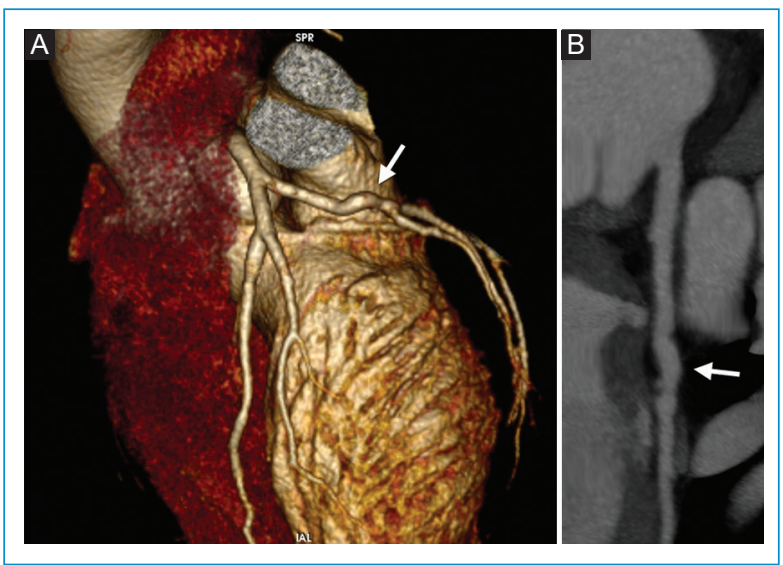

Figura 2. Angiotomografía de arterias coronarias A: reconstrucción volumétrica tridimensional que demuestra ectasia de la ACX proximal (flecha). B: reconstrucción curva que demuestra la extensión longitudinal y el aumento de calibre por ectasia de la ACX proximal (flecha).

El electrocardiograma y la ecocardiografía transtorácica fueron también normales, pero la prueba de esfuerzo con ejercicio se detuvo por presencia de dolor torácico en la quinta etapa del protocolo de Bruce, aunque sin cambios electrocardiográficos. Posteriormente, se realizó angiotomografía de arterias fusiforme proximal en la arteria coronaria derecha (ACD) de $11 \mathrm{~mm}$ de diámetro, con calcificación periférica y trombosis oclusiva. Igualmente, se identificó ectasia con calcificación en el tercio medio de la arteria circunfleja (ACX), en este caso sin trombosis (Figs. 1 y 2). Con estos hallazgos clínicos e imagenológicos el diagnóstico principal fue el de secuelas de enfermedad de Kawasaki. Para evaluar el flujo distal y determinar si el paciente se beneficiaba de una revascularización, se realizó coronariografía en la que se evidenció obstrucción completa de la ACD en su tercio proximal y presencia de flujo distal por circulación colateral heterocoronaria (Fig. 3). Debido a las características angiográficas, se decidió ofrecer manejo médico con antiagregación y anticoagulación para posteriormente intentar reperfundir utilizando técnicas para oclusión crónica.

\section{Discusión}

La enfermedad de Kawasaki es un síndrome febril agudo y autolimitado que afecta principalmente a menores de 5 años $^{1}$. Se trata de una vasculitis aguda de pequeño y mediano vaso que fue descrita por Tomisaki Kawasaki en $1967^{2}$. Su diagnóstico es desafiante, pero cobra gran importancia en pacientes jóvenes con eventos coronarios agudos debido a que, a pesar de su baja 


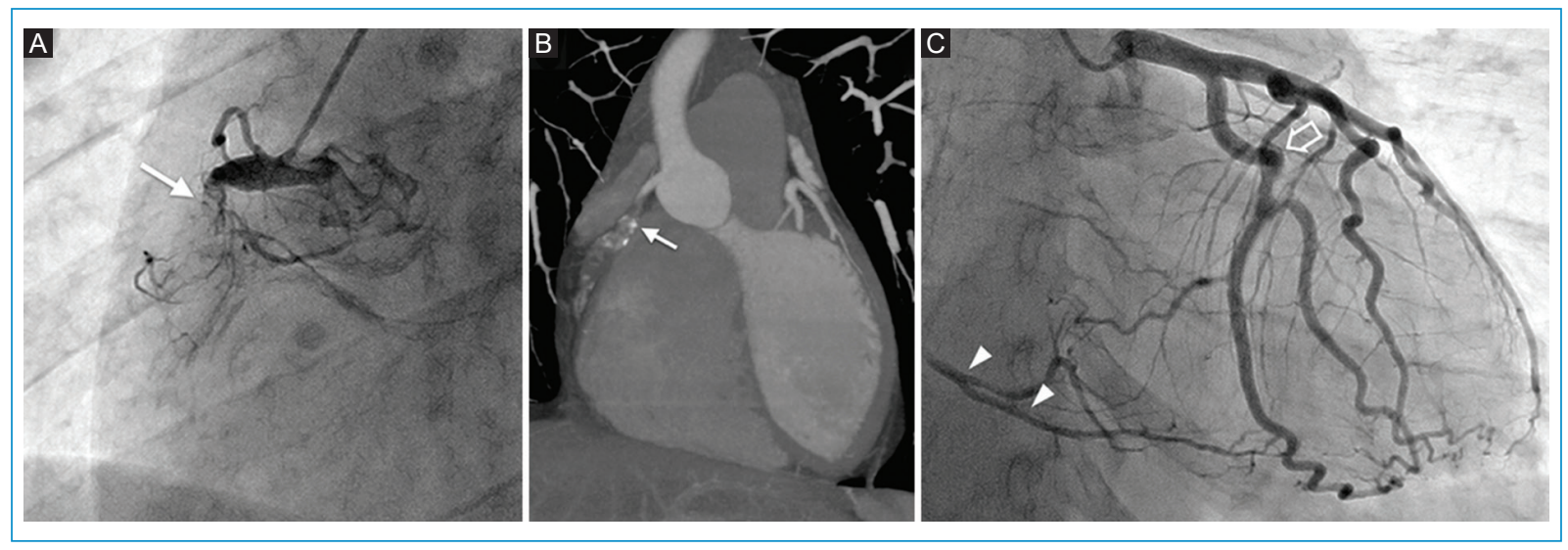

Figura 3. Coronariografía. A: ACD con dilatación proximal (flecha), calcificaciones periféricas y oclusión completa. No hay llenado distal. B: comparación con reconstrucción MPR de angiotomografía, con la que se obtiene mayor detalle de la anatomía e información extraluminal. C: tronco principal y ramas con permeabilidad, ectasia de la ACX (y circulación colateral hacia las ramas distales de la ACD (cabezas de flecha).

frecuencia, puede tener manifestaciones clínicas graves cuyo componente principal son alteraciones del sistema cardiovascular, específicamente de la circulación coronaria ${ }^{3}$. Su prevalencia e incidencia son más elevadas en países asiáticos, siendo Japón el sitio con mayor prevalencia. Esta tendencia se mantiene incluso en aquellos pacientes con ascendencia oriental que viven en países occidentales $^{2}$. Un fenómeno interesante que se describe con la enfermedad de Kawasaki es que muchos de los pacientes afectados durante su infancia llegarán a la adultez y se estima que su prevalencia podría aumentar hasta 1 por cada 1.600 adultos para el año 20304.

Su patogénesis es poco conocida, pero se presume una etiología autoinmune desenmascarada por una infección que lleva a un proceso inflamatorio persistente que predispone a remodelamiento coronario, disfunción endotelial, proliferación intimal y calcificación densa. Sumado a lo anterior, hay destrucción de la túnica media, lo cual favorece la formación de aneurismas ${ }^{5}$.

El diagnóstico es esencialmente clínico y no hay ningún biomarcador específico. Clásicamente ha sido considerado un síndrome febril con una duración mayor a 5 días sin un claro foco infeccioso y la presencia de al menos cuatro de cinco hallazgos clínicos característicos, tales como erupción polimórfica, inyección conjuntival, linfadenopatía cervical, eritema orofaríngeo y/o edema con descamación en manos y pies. Sin embargo, debe resaltarse que los criterios anteriores aplican sólo para la población pediátrica y específicamente para la fase aguda ${ }^{2}$. La fase crónica de la enfermedad generalmente es diagnosticada al documentar las complicaciones vasculares. La importancia de la identificación apropiada de la enfermedad de Kawasaki en su fase aguda radica en que muchos de los casos de infartos fatales y no fatales en personas jóvenes ( $<40$ años) sin otros factores de riesgo cardiovasculares, se han atribuido a secuelas de episodios agudos no diagnosticados ni tratados en la niñez. Estos eventos isquémicos parecen tener un comportamiento bimodal, presentándose a los dos años del episodio agudo y posteriormente en la tercera o cuarta décadas de la vida ${ }^{6}$. Esta relación se ha establecido gracias a los hallazgos angiográficos compatibles con este diagnóstico en este subgrupo de pacientes ${ }^{6,7}$.

En cuanto a la vasculatura coronaria, el compromiso más frecuente son los aneurismas, los cuales son usualmente proximales y comprometen la arteria descendente anterior y coronaria derecha. Los aneurismas se definen de acuerdo con las dimensiones coronarias ajustadas a la superficie corporal en la población pediátrica (parámetro denominado Z score), pero en adultos el diámetro del vaso es quizá el abordaje más adecuado. Un aneurisma con un diámetro mayor a $4 \mathrm{~mm}$ será definido como pequeño, entre 4 y $8 \mathrm{~mm}$ como mediano y mayor a $8 \mathrm{~mm}$ como grande/gigante ${ }^{1}$. Esta última estratificación es relevante debido a que la tasa de regresión de las dilataciones se relaciona directamente con el tamaño, siendo infrecuente en aquellos aneurismas gigantes, pero puede evidenciarse entre el 50 al $67 \%$ de los aneurismas pequeños o medianos en un periodo aproximado de uno a dos años ${ }^{8}$. A pesar de la pseudonormalización observada, esto no implica resolución completa de la enfermedad dado que estos pacientes continúan con riesgo aumentado de eventos cardiovasculares mayores de 
acuerdo con lo reportado en diferentes series ${ }^{9}$. Otros hallazgos imagenológicos frecuentes son las calcificaciones focales, circunferenciales y excéntricas, que se asocian, en la mayoría de los casos, a trombosis del vaso ${ }^{10}$. También se ha descrito la ectasia coronaria (hallazgo muy característico) y las estenosis focales, siendo estas últimas un vestigio de aquellas áreas en las que se presentó regresión espontánea de un aneurisma o una lesión ectásica ${ }^{11}$.

El compromiso vascular cardíaco es el sistema más afectado. Una serie francesa, en la que se reportaron 42 casos de enfermedad de Kawasaki del adulto, describió que el $44 \%$ de los sujetos refirió alguna manifestación cardiovascular; entre ellas las más comunes fueron dolor torácico, síndrome de falla cardíaca e infarto agudo de miocardio. En concordancia con lo anterior, 11 pacientes presentaban evidencia de vasculitis coronaria y 8 aneurismas coronarios. Los métodos diagnósticos utilizados fueron angiografía coronaria (63\%), angiografía coronaria por tomografía (25\%) o ecocardiografía $(13 \%)^{3}$. Además de las alteraciones descritas en el árbol coronario, también pueden presentarse otras formas de afectación cardíaca, tales como miocarditis, miopericarditis, derrame pericárdico, arritmias e incluso muerte cardíaca súbita ${ }^{12}$. Así mismo, se han descrito complicaciones en otros sistemas, como gastrointestinal, hepático, renal, respiratorio y músculo-esquelético.

Los diagnósticos diferenciales de los aneurismas coronarios engloban los originados por enfermedad aterosclerótica, los aneurismas congénitos y los inducidos por cocaína. La aterosclerosis es la causa más común de aneurismas coronarios (cerca del $50 \%$ ). Los aneurismas congénitos se presentan entre el 1 al $30 \%$ de los pacientes y generalmente se asocian con otras anormalidades, como fístulas en las arterias coronarias. Los aneurismas inducidos por abuso de cocaína cada vez son más comunes y en la actualidad su incidencia en los consumidores crónicos puede ser tan alta como del $30 \%$, y afectar predominantemente a pacientes menores de 44 años ${ }^{10}$.

El seguimiento dependerá de dos factores: el tamaño de los aneurismas y si el paciente ha desarrollado complicaciones, principalmente enfermedad isquémica del corazón. Las guías de la Sociedad Americana del Corazón (AHA, su sigla en inglés), recomiendan el uso de métodos diagnósticos no invasivos como alternativa a la coronariografía, dado que permiten evaluar la luz y la pared del vaso, una vez han pasado los periodos de dilatación y pseudonormalización ${ }^{1}$. Tanto la angiotomografía de arterias coronarias como la resonancia magnética cardíaca, han sido validadas en esta población. La primera ofrece mayor resolución espacial para la identificación y caracterización de las alteraciones vasculares y pueden aplicarse técnicas de baja dosis de radiación, como la adquisición prospectiva gatilla$\mathrm{da}^{13}$. La segunda, es útil en el seguimiento y la evaluación morfológica detallada en búsqueda de fibrosis y otras alteraciones cardíacas asociadas ${ }^{14}$.

El tratamiento ideal para la fase aguda de la enfermedad de Kawasaki consiste en la administración de inmunoglobulina intravenosa (2 $\mathrm{g} / \mathrm{kg}$ dosis única) y ácido acetilsalicílico (50-100 mg/kg/día dividida en cuatro dosis), antes del décimo día contado a partir del inicio de la enfermedad. Este tratamiento debe mantenerse hasta que se logre controlar el proceso inflamatorio (pseudonormalización del aneurisma) $)^{15}$. Con este esquema se logra disminuir la incidencia de complicaciones tardías, específicamente aneurismas coronarios, de un 25 a un $5 \%{ }^{16}$. El tratamiento de las complicaciones cardíacas (fase crónica), no está bien establecido en la literatura debido a falta de evidencia sólida, y se basará fundamentalmente en la gravedad de estas. La guía publicada por la AHA para enfermedad de Kawasaki, plantea continuar con ácido acetil-salicílico hasta que se resuelva el proceso inflamatorio; sin embargo, no hay información clara respecto a cuál es el momento más apropiado para suspenderlo y por ello algunos autores plantean continuar con antiagregación indefinida en aquellos con aneurismas $\geq 4 \mathrm{~mm}^{6}$. La anticoagulación debe contemplarse en aquellos casos de aneurismas gigantes o si se documenta trombosis. Finalmente, debido a la gran disfunción endotelial que muestran estos pacientes, podría contemplarse la administración de estatinas, pero no se tiene clara la población específica que se beneficiará, la dosis a prescribir y su duración ${ }^{17}$. Actualmente, está en curso un estudio sobre la utilidad de la atorvastatina en pacientes pediátricos con enfermedad de Kawasaki ${ }^{18}$. La revascularización coronaria se reserva para aquellos pacientes con síntomas anginosos de difícil control o síndromes coronarios agudos, y puede ser percutánea o quirúrgica. Una característica particular de estos pacientes es que tienen un alto riesgo de requerir múltiples procedimientos a lo largo de sus vidas ${ }^{15}$.

\section{Discusión}

Se presenta el caso de un paciente joven, con síntomas de angina, pero con bajo riesgo cardiovascular, en quien, a través de angiotomografía de coronarias, se detectaron signos radiológicos compatibles con 
secuelas de enfermedad de Kawasaki. Hasta el momento, consideramos se trata del primer reporte de las secuelas cardíacas por enfermedad de Kawasaki en un adulto en nuestro medio. El paciente mencionado cumple con varias de las características usualmente descritas en esta población, como dolor torácico como síntoma cardinal, inicio de los síntomas en la tercera década de la vida, ausencia de otros factores de riesgo cardiovascular y compromiso aneurismático proximal en la ACD con calcificaciones circunferenciales y periféricas en ausencia de cambios ateroscleróticos coronarios. Además, en este caso se identifican dos factores que podrían considerarse de mal pronóstico, como la trombosis completa del vaso y el diámetro de este (aneurisma gigante) y que indicarán el uso de anticoagulación.

Otro aspecto para resaltar es la utilidad de la angiotomografía como técnica de elección en la valoración de arterias coronarias en pacientes de riesgo bajo o intermedio, ya que permite la evaluación luminal, así como de la pared y de las cavidades cardiacas. Actualmente, se puede obtener una dosis de radiación equiparable o incluso menor que la angiografía convencional gracias a las técnicas de baja dosis (sincronización prospectiva).

\section{Conclusión}

La enfermedad de Kawasaki es una vasculitis que afecta característicamente la población infantil. Sin embargo, las complicaciones a mediano y largo plazo se presentan fundamentalmente en adultos sin factores de riesgo y por lo demás sanos, lo cual impone un reto a los cardiólogos de adultos y debe estar siempre presente en el diagnóstico diferencial de aquellos jóvenes que consulten por dolor torácico.

\section{Financiamiento}

Los autores notifican no haber recibido ningún tipo de remuneración o soporte económico por la publicación del presente artículo.

\section{Conflicto de intereses}

Los autores del presente documento declaran no presentar conflicto de intereses.

\section{Responsabilidades éticas}

Protección de personas y animales. Los autores declaran que para esta investigación no se han realizado experimentos en seres humanos ni en animales.

Confidencialidad de los datos. Los autores declaran que han seguido los protocolos de su centro de trabajo sobre la publicación de datos de pacientes.

Derecho a la privacidad y consentimiento informado. Los autores han obtenido el consentimiento informado de los pacientes y/o sujetos referidos en el artículo. Este documento obra en poder del autor de correspondencia.

\section{Bibliografía}

1. McCrindle BW, Rowley AH, Newburger JW, Burns JC, Bolger AF, Gewitz M, et al. Diagnosis, treatment, and long-term management of Kawasaki disease: A scientific statement for health professionals from the American Heart Association. Circulation. 2017;135:927-99.

2. Kontopoulou T, Kontopoulos DG, Vaidakis E, Mousoulis GP. Adult Kawasaki disease in a European patient: A case report and review of the literature. J Med Case Rep. 2015;9(1):1-7.

3. Fraison JB, Sève P, Dauphin C, Mahr A, Gomard-Mennesson E, Varron L, et al. Kawasaki disease in adults: Observations in France and literature review. Autoimmun Rev. 2016;15(3):242-9.

4. Huang S-K, Lin MT, Chen HC, Huang SC, Wu MH. Epidemiology of Kawasaki Disease: Prevalence from National Database and Future Trends Projection by System Dynamics Modeling. J Pediatr. 2013;163(1):126-31.e1.

5. Shulman ST, Rowley AH. Kawasaki disease: Insights into pathogenesis and approaches to treatment. Nat Rev Rheumatol. 2015;11(8):475-82.

6. Denby KJ, Clark DE, Markham LW. Management of Kawasaki disease in adults. Heart. 2017;103(22):1760-9.

7. Burns JC, Shike H, Gordon JB, Malhotra A, Schoenwetter M, Kawasaki T. Sequelae of Kawasaki disease in adolescents and young adults. J Am Coll Cardiol. 1996;28(1):253-7.

8. Manlhiot C, Niedra E, McCrindle BW. Long-term management of Kawasaki disease: Implications for the adult patient. Pediatr Neonatol. 2013;54(1):12-21.

9. Iemura M, Ishii M, Sugimura $T$, Akagi $T$, Kato $H$. Long term consequences of regressed coronary aneurysms after Kawasaki disease: Vascular wall morphology and function. Heart. 2000;83(3):307-11.

10. White CS, Kligerman SJ, Killam JL, Jacob W, Shah AB, Frazier AA. Spectrum of Coronary Artery. RadioGraphics. 2018;38(1):11-6.

11. Duarte R, Cisneros S, Fernandez G, Castellon D, Cattani C, A Melo C, et al. Kawasaki disease: a review with emphasis on cardiovascular complications. Insights Imaging. 2010;1(4):223-31.

12. Garg J, Krishnamoorthy P, Palaniswamy C, Paudel R, Chatterjee S, Ahmad $\mathrm{H}$, et al. Prevalence and predictors of coronary artery disease in adults with Kawasaki disease. Cardiol Young. 2014;25(6):1124-9.

13. Singhal M, Gupta $P$, Singh S, Khandelwal N. Computed tomography coronary angiography is the way forward for evaluation of children with Kawasaki disease. Glob Cardiol Sci Pract. 2017;2017(3):e201728. doi: $10.21542 /$ gcsp.2017.28

14. Greil GF, Stuber M, Botnar RM, Kissinger KV, Geva T, Newburger JW, et al. Coronary magnetic resonance angiography in adolescents and young adults with Kawasaki disease. Circulation. 2002;105(8):908-11.

15. JCS Joint Working Group. Guidelines for diagnosis and management of cardiovascular sequelae in Kawasaki Disease (JCS 2008). Circ J. 2010;74(9):1989-2020.

16. Daniels LB, Tjajadi MS, Walford HH, Jimenez-Fernandez S, Trofimenko V Fick DB, et al. Prevalence of Kawasaki disease in young adults with suspected myocardial ischemia. Circulation. 2012;125(20):2447-53.

17. Brogan P, Burns JC, Cornish J, Diwakar V, Eleftheriou D, Gordon JB, et al. Lifetime cardiovascular management of patients with previous Kawasaki disease. BMJ; 2020;106:411-20.

18. Tremoulet AH, Jain S, Burns JC. Evaluating a novel treatment for coronary artery inflammation in acute Kawasaki disease: A Phase I/lla trial of atorvastatin. Expert Opin Orphan Drugs. 2015;3(9):967-70. 